\title{
Getting Around in Google Cardboard - Exploring Navigation Preferences with Low-Cost VR
}

\author{
Wendy Powell ${ }^{\star}$, Vaughan Powell, Phillip Brown, Marc Cook, Jahangir Uddin \\ University of Portsmouth
}

\begin{abstract}
In recent years there has been a paradigm shift in the uptake and use of Virtual Reality (VR). Advances in graphics rendering, and the introduction of low-cost VR headsets has brought VR into the reach of ordinary consumers. Google Cardboard VR viewers cost just a few dollars and work with most smart phones, enabling VR to truly enter the domain of the everyday. However, these headsets are currently generally used for passive entertainment or viewing 360 degree media, and are not ideally suited to active exploration of a virtual space. In this paper we present our preliminary evaluation of three approaches to travel and navigation.
\end{abstract}

Keywords: Interaction, travel techniques, HMD, mobile VR, Google Cardboard, navigation

Index Terms: I.3.6 [Methodology and techniques]: Interaction techniques; H.5.2 [User interfaces]: Input devices and strategies

\section{BACKGROUND}

In the past, Virtual Reality (VR) has been a specialist domain outside the reach of most ordinary consumers [1]. The cost and space requirements for immersive VR applications, together with the technical limitations, have prevented widespread uptake at a consumer level. However, technical developments in recent years, combined with a resurgence of interest in Virtual Reality has brought it into the reach of the general public, and portable or built-in tracking capability has given VR the potential to become a truly everyday commodity.

Recent advances in mobile graphics have given modern Smartphones' capabilities which surpass the desktop systems of just a few years ago [2]. In 2014, Google introduced the concept of "Cardboard VR" - virtual reality headsets costing just a few dollars, and working with most modern mobile phones [3]. Whilst they are not intended to compete in the market with offerings such as Oculus Rift [4] or Samsung Gear VR [5], the ultra-low cost makes them attractive to those who may not otherwise considering entering the VR market. By January 2016, there were 5 million users of Cardboard VR, with over 1000 apps available [6]. However, there are a number of limitations with entry-level VR which restrict its scope of application. The field of view (FOV) is somewhere between $60^{\circ}$ and $100^{\circ}$, depending on the specific phone used in the headset. Graphical fidelity is inevitably lower than that which can be produced using the computing power of a PC, and the lack of efficient cooling hardware in most phones means that they heat quickly with any application which requires continuous display of graphics.

\footnotetext{
*wendy.powell@port.ac.uk
}

Until recently, input was restricted to a single magnetic sliding switch [7], and head tracking relies on motion detected by the phone's built-in accelerometer or gyroscope [8].

Even with the release of Google Cardboard V2, which has a capacitive button instead of the magnetic switch, control options are still very limited, and at the present time they are used primarily for viewing 360 degree photos and videos, or for "passive entertainment" e.g. riding a virtual rollercoaster. Most current applications are constrained by the limited number of interaction possibilities. Active selection of menus and other objects can be achieved by "gaze selection" techniques (the user looks directly at the desired object for an extended period of time [9]), or by a switch on the side of the headset [7]. Neither of these techniques is conducive to active exploration of a virtual space. However, for most VR applications, navigation is a core task [10], and consideration should be given as to how best support navigation techniques in these emerging devices The most common approaches include fixing the user in one place and simply allowing them to look around (most $360^{\circ}$ videos and scenes use this approach), or to move them continually forwards on an animation path, or in the direction of gaze, stopping only when colliding with virtual objects. None of these approaches facilitates user-controlled exploration of the virtual world. Furthermore, there have not yet been any user studies evaluating the pros and cons of these approaches, or exploring alternatives.

Whilst there is a substantial body of HCI research exploring navigation in 2D and 3D interfaces, many of the approaches are unsuitable for use with Google Cardboard. Wide area tracking and other vision-based techniques rely on external equipment which is the antithesis of mobile VR. Likewise, keyboard and mouse input requires an external computer, which negates the purpose of rendering straight to the phone. A joystick or game controller has some potential, as newer models offer a Bluetooth connection which can communicate directly with a mobile phone. However, these are generally fairly bulky, and don't really facilitate truly portable everyday VR. The 3D user interface community needs to look beyond traditional input techniques in order to provide acceptable navigation in mobile VR.

Bowman [10] divides navigation tasks into the components of way-finding and travel, and it is this latter component which is the focus of this paper. At its core, travel involves the movement of the viewpoint within the virtual environment from one place to another. This movement can be instantaneous or it can involve both temporal and spatial components. For the purpose of this study, we will be using a steering metaphor, with continuous specification of direction using head tracking. Travel will be restricted to the $\mathrm{x}-\mathrm{z}$ plane to reduce cognitive load and improve usability [11].

\section{Methodology}

The great appeal of Google Cardboard is its accessibility. It works anywhere, with almost any phone, and is lightweight and portable. Therefore we wanted to focus this preliminary evaluation on techniques which supported this level of ultra-low 
cost portable VR. Although some authors recommend separating the viewing direction from the travel direction [12], this necessitates an additional input in order to set a travel direction which is independent of the viewpoint orientation. As the goal of this study is to evaluate techniques using minimal input, we implemented travel in the direction of gaze.

The simplest headset simply holds the phone, with no additional controls, and this was used as the baseline for our first technique. Many entry-level headsets include a sliding magnetic switch which can be detected by the magnetometer in a smart phone, and so for the second technique we added a stop-start toggle functionality controlled by the sliding magnet. Finally, some lowcost headsets come supplied with a small Bluetooth controller, and we used the mini-joystick to allow direct control of forward and backward movement only (Table 1). Conditions were presented in counterbalanced order, to minimise any order effect.

Table 1: The three travel techniques evaluated in the study

\begin{tabular}{|l|l|}
\hline Condition & $\begin{array}{l}\text { Level of control } \\
\text { (travel is in the direction of gaze) }\end{array}$ \\
\hline Continuous motion & No control of travel \\
\hline Magnetic switch & $\begin{array}{l}\text { Travel can be stopped and started using } \\
\text { toggle switch }\end{array}$ \\
\hline Bluetooth controller & $\begin{array}{l}\text { Direct control of forward and backward } \\
\text { travel }\end{array}$ \\
\hline
\end{tabular}

Navigation tests for evaluation of travel techniques should compel the user to perform both simple and complex navigation tasks [13]. In order to achieve this, we planned a route which required navigation in and out of two doorways, and a number of changes of direction, as well as turning in both constrained and open spaces (Figure 1).

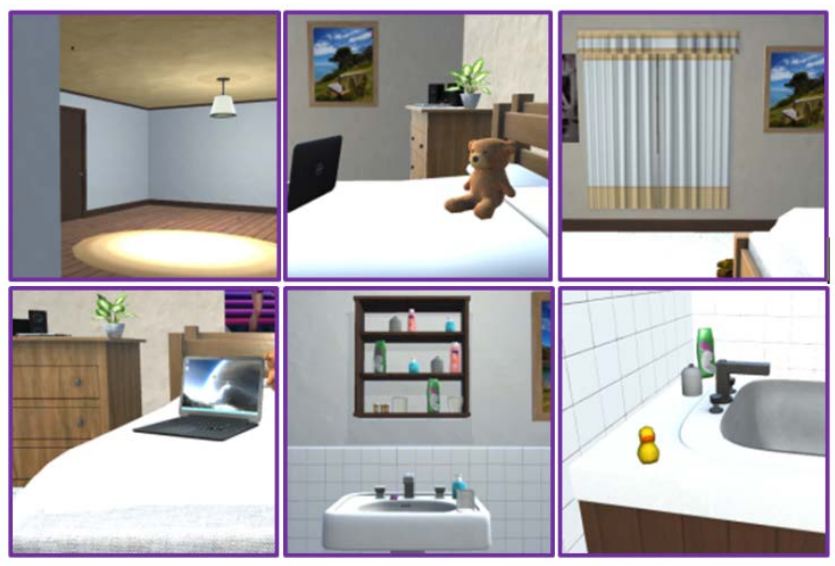

Figure 1: The six target locations visited in the study

\subsection{Equipment}

A virtual flat was built in 3DS max and deployed in the Unity game engine, using the Google Cardboard virtual camera as the viewer. For each technique, the movement was set at the same (steady walking) speed of $1.5 \mathrm{~m} / \mathrm{s}$.

The application was deployed as an .apk file onto a Nexus 6 mobile phone, and an operator view was mirrored in real-time on a standard PC laptop. The phone was mounted inside a DeFairy VR headest. The controller used was a DeFairy mini bluetooth controller, mapped to allow only forward and backward movement using the mini joystick. Participants were seated throughout the tasks on a swivel chair with armrests (Figure 2).

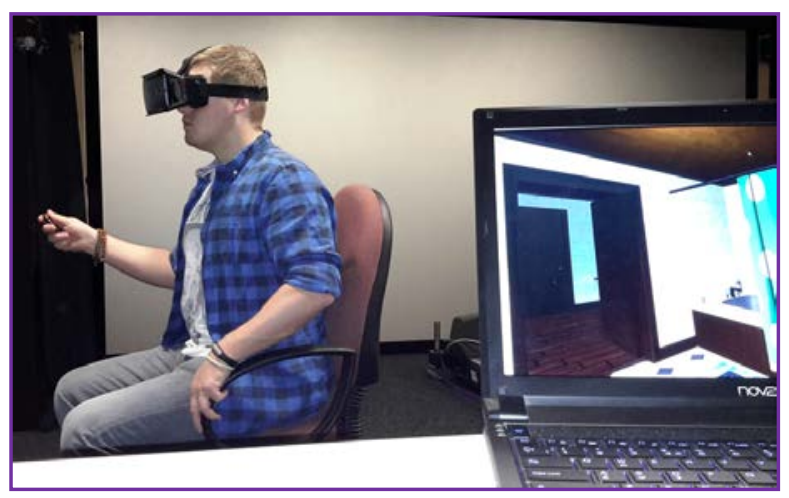

Figure 2: The experimental setup

\subsection{Procedure}

The navigation task was designed to visit six locations in sequence (Figure 1). The target locations were selected to provide consistency of experience between tasks, and to ensure that participants were required to change direction and manoeuvre sufficiently to test both simple and complex navigation [13].

Participants were briefed on the task and the techniques, and given time to familiarise themselves with the virtual environment using keyboard controls on the laptop computer. They then completed the navigation task using the VR headset three times, once for each travel technique, and answered a short series of questions after each trial. As the study did not involve memory or cognition testing, each time the participants reached a target location they were given a verbal prompt to direct them to the next location.

Each trial was timed from the start of movement until returning to the hallway at the end of the trial. In order to record natural navigation behaviour, participants were not informed that they were being timed.

\section{Results}

There were three types of observations recorded during this study which will be considered in separate sections.

\subsection{Time to complete the navigation task}

A repeated measures one-way ANOVA demonstrated a significant effect of travel technique on task completion time $\left(\mathrm{F}_{2,11}=7.25, \mathrm{p}<0.01\right)$. The mean completion time was fastest with the Bluetooth controller (50s), and slowest with the magnetic switch (85s) (Figure 3).

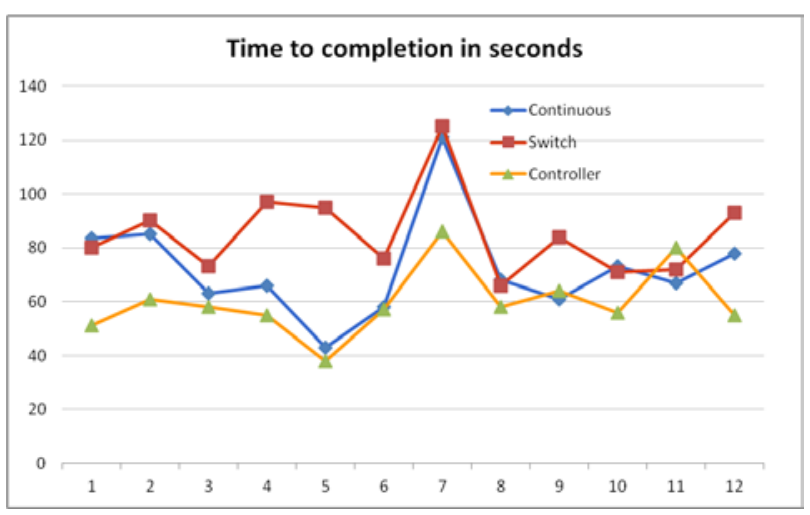

Figure 3: Task completion time per user for each travel technique 


\subsection{User experience scores}

Participants were asked three questions after each technique (Table 2). Each was scored on a Likert scale from 1-5.

\begin{tabular}{|l|l|l|l|}
\hline & Continuous & Switch & Controller \\
\hline $\begin{array}{l}\text { Ease of movement } \\
\text { (1=easy, 5=hard) }\end{array}$ & 2.9 & 2.1 & 1.4 \\
\hline $\begin{array}{l}\text { Sense of presence } \\
\text { (1=real, 5=unreal) }\end{array}$ & 2.8 & 2.9 & 2.8 \\
\hline $\begin{array}{l}\text { Liked the technique } \\
\text { (1=liked, 5=disliked) }\end{array}$ & 3.0 & 2.3 & 1.8 \\
\hline
\end{tabular}

Table 2: Mean Likert score for each question

A repeated measures one-way ANOVA demonstrated a significant effect of travel technique on the participants perceived ease of use $\left(\mathrm{F}_{2,11}=8.36, \mathrm{p}<0.01\right)$.

A repeated measures one-way ANOVA demonstrated a significant effect of travel technique on enjoyment of the technique $\left(\mathrm{F}_{2,11}=3.95, \mathrm{p}<0.05\right)$.

There was a strong positive correlation between perceived ease of movement, and enjoyment of the technique $(r=0.80)$.

There was no significant effect of travel technique on the minipresence score ("Felt like I was really in the flat").

There was no correlation between perceived ease of use, and task completion times $(\mathrm{r}=0.20)$.

At the end of the study, participants were asked to reflect on all three techniques and to compare them. The Bluetooth controller was the preferred travel technique, which was also expressed to be the easiest to use. The continuous motion was both the least liked technique, and the one that participants felt was hardest to use (Figure 4).

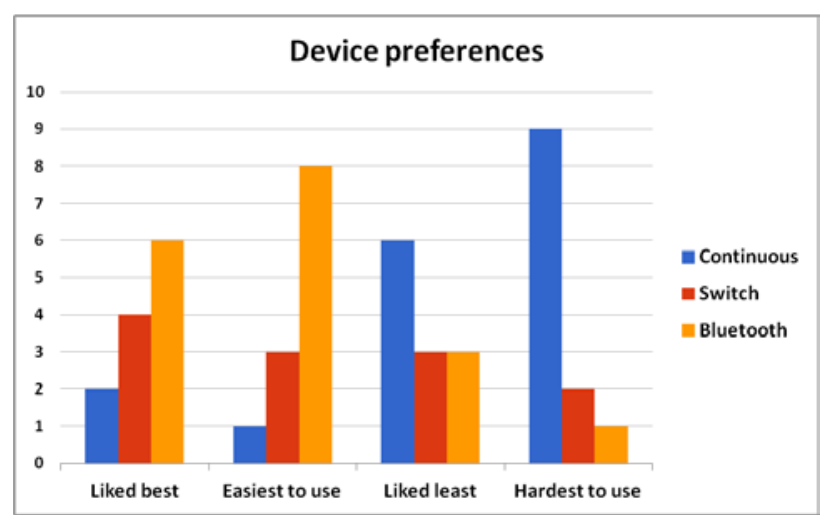

Figure 4: Task completion time per user for each travel technique

\subsection{Qualitative feedback}

After each trial, and at the end of the study, participants were given an opportunity for additional comments on the travel technique. The most frequent comments fell into the broad categories of "control" and "naturalness".

\subsubsection{Perception of control of movement}

Participants generally disliked the lack of control of movement, particularly during the continuous motion technique. This was mostly related to the need to plan movement ahead, the inability to turn tightly while moving forward, and being unable to stop accurately at target locations. The Bluetooth controller gave a much greater sense of control, and required less movement planning. Some participants observed that the continuous movement was quite good for exploration of larger areas, but not for local manoeuvring. The magnetic switch had potential, but didn't always respond on first click, and was harder to stop at a precise point. Interestingly, most participants felt that this technique was easier than the continuous motion, but this did not correlate to their task completion times, which were faster with continuous motion than with the switch even though they had to correct errors. A number of participants expressed a desire for speed control (acceleration) across all three techniques, and for the ability to strafe (side-step) when using the controller.

\subsubsection{Naturalness of movement}

Although there was no explicit recording of gaming experience, several participants commented that their previous gaming experience made the joystick control intuitive. However, others expressed that it produced a disconnect between the control and the environment. For manoeuvring, the continuous movement technique required movement planning, particularly when there was no switch control. However, many participants felt that not having to explicitly think about movement control gave a more natural experience, described as "immersive", "visceral" or "quite pleasant". Indeed, many users were observed to engage their whole body when using these techniques, leaning and tilting in an attempt to make their physical movements support the virtual travel, indicative of a higher level of immersion. This behaviour was not evident when using the handheld controller. The restrictions in looking around while moving were problematic for some, giving a sense of reduced peripheral vision.

\section{Discussion}

The performance and graphical fidelity of Google Cardboard techniques cannot compete with more expensive VR solutions, but it has the huge advantage of allowing the wider general public to access VR with minimal financial outlay. The quality and enjoyment of these first experiences could, for many people, significantly influence their desire to engage with VR in the future. Therefore, rather than dismissing this entry level VR as a cheap gimmick, irrelevant to serious aficionados, we should be actively working to ensure that this everyday VR is as enjoyable and easy to use as possible.

Whilst the findings of this preliminary work should be interpreted with caution, they do give some useful insight into the user experience, and offer some guidelines which may be useful to consider when designing applications for everyday VR.

First and foremost, it is important to remember that we may have little control over the type of headset which is being used. The default position is likely therefore to involve continuous movement, perhaps mediated by gaze-directed control or other software-mediated device. In this situation, any requirement to make tight turns or unplanned manoeuvres should be avoided, and a suitable collision boundary provided at points where the user may wish to stop. Whilst in ideal circumstances users should be provided with an independent line of site without changing direction $[12,14]$, this may prove difficult when input options are limited.

Bluetooth controllers are available at very low cost, and indeed are often included with the purchase of a headset. For many users, particularly gamers, this provides a natural control, but it was reported to create a disconnect between the user and the virtual environment. However, this method was the most efficient for task performance, being nearly 50\% faster than the magnetic switch control. This result is likely to vary with different navigation tasks, but is a significant factor, particularly where efficient manoeuvring is required in an unfamiliar environment. 
The addition of the switch control to the continuous movement increased the time taken to complete the task without greatly improving the user experience, However, the current magnetic switches are not $100 \%$ reliable [7], and it may be that a more responsive switch might bridge the gap between the immersiveness of continuous movement and the ease of use of the handheld controller. With the release of the V2 headset it will be interesting to evaluate whether the new capacitive switch will impact these findings. It was noteable that this switch technique was significantly slower than the less controlled continuous motion. Whilst we might have anticipated fewer errors and corrections of direction, this did not seem to be the case, and, furthermore, most users chose to stop and turn at almost every location, even if they had previously navigated them smoothly using continuous motion.

In contrast to reported immersion scores, we observed that the physical behaviour of the participants indicated a higher level of immersion when using the continuous controls than with the Bluetooth controller, and this warrants further investigation as it may have implications for certain types of application. Where increase immersion is desirable, then a hand-held controller may detract from this. However, the body motions, both forward and back and laterally, may increase the risk of injury or falls, and this is a significant consideration in unsupervised everyday VR.

In summary, none of the three techniques evaluated in this study offered an ideal solution for travel within VR, but a continuous motion with the ability to control the speed and stop would seem to be the best compromise. Further work is needed to explore these and other techniques in more depth, and this should be an area for fruitful discussion in the WEVR workshop.

\section{ACKNOWLEDGEMENTS}

With thanks to Mark Silvester for his contributions to the 3D model of the virtual flat.

\section{REFERENCES}

[1] Amer, A. and P. Peralez. Affordable altered perspectives: Making augmented and virtual reality technology accessible. in Global Humanitarian Technology Conference (GHTC), IEEE. 2014.

[2] Steed, A. and S. Julier. Design and implementation of an immersive virtual reality system based on a smartphone platform. in 3D User Interfaces (3DUI), 2013 IEEE Symposium on. 2013.

[3] Google, Google Cardboard VR. URL: https://www.google.com, 2014.

[4] Oculus, Oculus rift-virtual reality headset for 3d gaming. URL: http://www. oculusvr. com, 2012.

[5] Samsung, Samsung Gear VR. URL: https://www.oculus.com/enus/gear-vr/, 2015.

[6] Google, (Un)folding a virtual journey with Google Cardboard, in Google Official Blog. 2016.

[7] Smus, B. and C. Riederer, Magnetic input for mobile virtual reality, in Proceedings of the 2015 ACM International Symposium on Wearable Computers. 2015, ACM: Osaka, Japan. p. 43-44.

[8] Sharma, P., Challenges with virtual reality on mobile devices, in ACM SIGGRAPH 2015 Talks. 2015, ACM: Los Angeles, California. p. 1-1.

[9] Sibert, L.E. and R.J.K. Jacob, Evaluation of eye gaze interaction, in Proceedings of the SIGCHI conference on Human Factors in Computing Systems. 2000, ACM: The Hague, The Netherlands. p. 281-288.

[10] Bowman, D.A., et al., 3D User Interfaces: Theory and Practice. 2004: Addison Wesley Longman Publishing Co., Inc.
[11] Sebok, A., E. Nystad, and S. Helgar, Navigation in desktop virtual environments: an evaluation and recommendations for supporting usability. Virtual Reality, 2004. 8(1): p. 26-40.

[12] Bowman, D.A., R.P. McMahan, and E.D. Ragan, Questioning naturalism in 3D user interfaces. Commun. ACM, 2012. 55(9): p. 7888.

[13] Sousa Santos, B., et al., Head-mounted display versus desktop for 3D navigation in virtual reality: a user study. Multimedia Tools and Applications, 2008. 41(1): p. 161-181.

[14] Sayers, H., et al. Usable interfaces for virtual environment applications on non-immersive systems. in Proceedings of the 18th Annual Eurographics UK Conference, Swansea. 2000. 